\title{
Dernières nouvelles
}

Julia Vecsey

Correspondance:

Dr Julia Vecsey

Quai Gustave-Ador 62

CH-1207 Genève

jvecsey[at]bluewin.ch
L'an dernier, j'ai trouvé lors d'une promenade un appareil de photo argentique abandonné sur un banc public. Dévoré par la curiosité, j'ai alors entrepris de faire développer la pellicule qu'il contenait. Il s'est avéré que ce n'était pas simple:

Il s'agissait tout d'abord de trouver une pile compatible avec l'appareil en question pour rembobiner la pellicule. Les temps changent et les magasins de photographies ne courent plus les rues... Une fois ce problème résolu, il fallait encore faire développer le tout, même combat!

Et alors, oh! surprises!

Parmi plusieurs images, je découvris une photographie d'un parking de Leicester, dans lequel des archéologues s'affairaient autour d'une fosse creusée au beau milieu du terrain. Cette étrange manœuvre avait permis aux scientifiques de découvrir des ossements humains prétendument protégé par une cuirasse moyenâgeuse. Visiblement, cette dernière n'avait pas réussi à sauver la vie du vaillant guerrier, puisqu'il était mort au combat, transpercé par une lance. L'ADN découvert correspondait à celui de Richard III, roi anglais que les historiens pensaient avoir été tué à cet endroit lors d'une bataille, raison pour laquelle ils avaient entrepris ces fouilles en ce lieu précis . Et bingo, en plein dans le mille!

Il était tombé de cheval lors du combat, et son animal avait été à l'origine d'une dynastie dont on a pu retrouver des traces dans des lasagnes six siècles plus tard...

Pour sauver la face de ce plat décrié, il a été accommodé par un grand cuisinier italien, et adapté de façon à améliorer le (pain) quotidien d'astronautes embarqués dans un futur vaisseau spatial pour gagner la planète mars, long voyage s'il en est.

Tout cela prouve bien que les êtres humains adorent la viande, et puisque nous sommes tellement nombreux sur terre, il faut essayer de trouver une alternative pour alimenter tout ce beau monde: on a parlé des insectes, des vers de terre, des serpents et autres limaces, solutions pas vraiment ragoûtantes, alors pourquoi ne pas essayer de confectionner des steaks in vitro! Si, si, ça existe, c'est pas très bon mais au moins ça a le mérite de nous revigorer.

Une autre image montrait de manière saisissante et inquiétante le repli de la neige: elle faisait partie d'un reportage photographique montrant sur deux photos comparatives, prises à 20 ans d'intervalle, un recul des glaciers de $200 \mathrm{~m}$.

Je me demandai alors à qui avait bien pu appartenir cet appareil. La réponse me fut donnée par le prochain cliché de la bobine: il s'agissait d'une prise de vue de l'entrée du journal la Suisse, disparu encore au $\mathrm{XX}^{\mathrm{e}}$ siècle, immortalisée par le photographe-reporter.
Alors, très satisfait au fond de moi-même d'avoir été en mesure de dater l'objet découvert, je réalisai que l'histoire n'était pas possible. La fonte des neiges par exemple n'était pas encore aussi rapide à la fin du $\mathrm{XX}^{\mathrm{e}}$ siècle. Nous n'étions pas aussi nombreux sur terre. Tout n'était pas encore aussi accéléré qu'aujourd'hui.

Alors il fallait bien se rendre à l'évidence, un autre que moi avait trouvé l'appareil, avait immortalisé des faits récents, puis, pour une raison inconnue, l'avait abandonné sur ce banc public...

Donc pour assembler le puzzle, je continuai à découvrir les images suivantes:

J'ai alors vu sur une plage d'Afrique du Nord des camions débordant de sable. Ce matériau volé est utilisé pour la construction de résidences de luxe, à l'aide d'un trafic incontrôlable et lucratif.

Ce même sable est parfois exporté par bateau vers les ports européens, donnant l'occasion à une foule de micro-organismes exotiques de déferler sur le vieux continent. Mais grâce à des capteurs qu'on peut ingérer et intégrer à nos organismes, nous avons les moyens d'anticiper la mise en route de défenses, que ce soit grâce à des antibiotiques ou à l'aide de venin de serpent.

Lorsque l'infection n'arrive pas par bateau, elle peut arriver par avion, pour autant qu'un nuage de cendres ne bloque pas la circulation aérienne comme après l'éruption du volcan islandais Eyjafjallajökull; à propos, pour qu'une telle mésaventure ne se reproduise pas, il est prévu d'organiser une surveillance par rayon laser au niveau européen.

Il y avait aussi sur la pellicule la photo d'un petit garçon; et celle de coupures de journaux racontant qu'un papa divorcé, frustré de ne pas être autorisé à voir son propre fils, avait fait le siège d'une grue, menaçant de mettre fin à ses jours si la justice ne lui rendait pas son droit de visite.

Alors, j'ai tout compris: le banc sur lequel j'avais trouvé l'appareil était situé au pied d'une grue, et donc le papa désespéré ne pouvait être autre que mon photographe. 